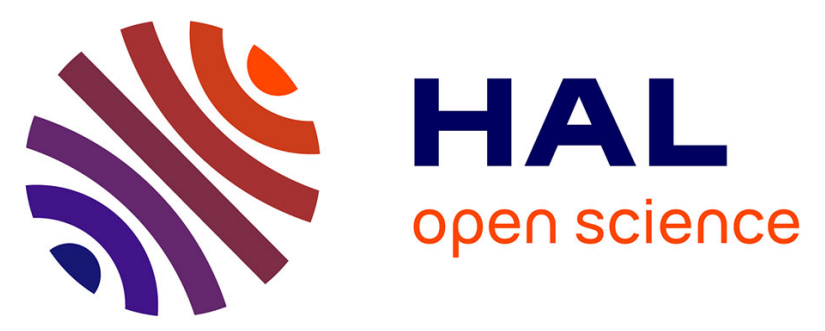

\title{
Determination of the full deformation tensor by multi-Bragg fast scanning nano X-ray diffraction
}

Andreas Johannes, Jura Rensberg, Tilman A. Grünewald, Philipp Schöppe, Maurizio Ritzer, Martin Rosenthal, Carsten Ronning, Manfred Burghammer

\section{- To cite this version:}

Andreas Johannes, Jura Rensberg, Tilman A. Grünewald, Philipp Schöppe, Maurizio Ritzer, et al.. Determination of the full deformation tensor by multi-Bragg fast scanning nano X-ray diffraction. Journal of Applied Crystallography, 2020, 53 (1), pp.99-106. 10.1107/S1600576719016534 . hal02466436

\section{HAL Id: hal-02466436 \\ https://hal-amu.archives-ouvertes.fr/hal-02466436}

Submitted on 4 Feb 2020

HAL is a multi-disciplinary open access archive for the deposit and dissemination of scientific research documents, whether they are published or not. The documents may come from teaching and research institutions in France or abroad, or from public or private research centers.
L'archive ouverte pluridisciplinaire HAL, est destinée au dépôt et à la diffusion de documents scientifiques de niveau recherche, publiés ou non, émanant des établissements d'enseignement et de recherche français ou étrangers, des laboratoires publics ou privés.

$$
\text { Copyright }
$$




\title{
Determination of the full deformation tensor by multi-Bragg fast scanning nano $\mathrm{X}$-ray diffraction
}

\author{
Andreas Johannes, ${ }^{a}$ Jura Rensberg, ${ }^{b}$ Tilman A. Grünewald, ${ }^{a, c}$ Philipp Schöppe, ${ }^{b}$ \\ Maurizio Ritzer, ${ }^{b}$ Martin Rosenthal, ${ }^{a}$ Carsten Ronning ${ }^{b}$ and Manfred \\ Burghammer $^{\mathrm{a} *}$
}

${ }^{\text {a} E}$ uropean Synchrotron Radiation Facility, 71 avenue des Martyrs, Grenoble, 38043, France, ${ }^{\mathbf{b}}$ Institut $\mathrm{f}$ r Festkörperphysik, Friedrich-Schiller-Universität Jena, Max-Wien-Platz 1, Jena, 07743, Germany, and Institut Fresnel, Avenue Escadrille Normandie Niemen, Marseille, 13013, France. *Correspondence e-mail: manfred.burghammer@esrf.fr

This work showcases a method to map the full deformation tensor in a single micro-sized crystal. It is shown that measuring the position of two Bragg reflections in reciprocal space is sufficient to obtain the full deformation tensor, if the condition of incompressibility of the material is imposed. This method is used to reveal the surface tension induced deformation at the edges of an asgrown single-crystal $\mathrm{VO}_{2}$ microwire. All components of the deformation tensor of the microwire were measured down to an absolute value of $10^{-4}$ in an $8 \times 14 \mu \mathrm{m}$ projected area of the wire. With a beam-defined spatial resolution of $150 \times 150 \mathrm{~nm}$, the measurement time was merely $2.5 \mathrm{~h}$.

\section{Introduction}

Internal stresses are an often neglected property of a given material. However, they can play a critical role in determining the material properties, allowing for often surprising changes to them. The most well known example must be Prince Rupert's drops (Aben et al., 2016), and also the properties of semiconductors, such as $\mathrm{Si}, \mathrm{SiGe}$ and Ge (Lee et al., 2005). In particular, phase-change materials, such as the $\mathrm{VO}_{2}$ (Liu et al., 2011) investigated here, can be significantly altered by external or internal stresses leading to strain. A very powerful method to measure strain in materials is X-ray diffraction (XRD). Due to the small wavelength and excellent monochromaticity of the available beams, XRD is a popular method for strain analysis.

In recent years synchrotron sources have become available to measure XRD down to a spatial resolution of tens of nanometres. At these nano-diffraction beamlines typically a sample is raster-scanned at multiple angles around the diffraction condition for a specific Bragg reflection, as identified by the Miller indices $(h k l)$. The Bragg reflection can thus be imaged in three dimensions for each point of the raster scan. Except when using advanced methods relying on the coherent properties of the beam (such as ptychography; Thibault et al., 2008), the position of the centre of mass of the Bragg reflection in reciprocal space is typically identified as the components of the scattering vector from the planes associated with $h k l$. The relative change in the length of the scattering vector is caused by the compressive/tensile strain in that direction. The direction of the scattering vector can be expressed as the pitch and yaw of the $h k l$ planes. Therefore, a relative change in these angles corresponds to shear in the respective direction of the planes. Unfortunately, the experimenter is completely blind to any strain and shear in the

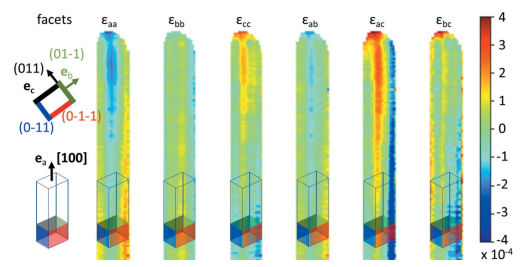

Edited by A. Borbély, Ecole National Supérieure des Mines, Saint-Etienne, France

Keywords: nano X-ray diffraction; deformation tensor; strain. 
remaining directions and must rely on symmetry arguments if the interesting property happens to include contributions from any other strain components. Alternatively, the same area can be scanned at a different orientation to map an additional Bragg reflection and gather the respective strain information for that direction. Finally, the information from both scans must be combined, which can become especially problematic considering that the projection along which the strain is probed changes for a different sample orientation.

Alternatively, strain measurements are conducted with 'white', i.e. broad-spectrum, X-ray beams. In these experiments it is usual that multiple Bragg peaks fulfil the diffraction condition and they are easily imaged. However, the photon energy in the diffracted beams is not known a priori and must be determined. Traditionally this was done with a monochromatic reference measurement (Chung \& Ice, 1999), which has been facilitated with the recently developed rainbowfiltering technique (Robach et al., 2013; Tardif et al., 2016). Ongoing developments of energy-dispersive detectors are also used in such strain mapping experiments. These are straightforward, but suffer from the inherently low count rate of monochromatic pixel detectors (Abboud et al., 2017). As outlined in the cited work and references therein, the polychromatic methods are very successful in imaging strain, including the full deformation tensor. However, compared with the monochromatic experiment outlined here, the use of a polychromatic beam has several disadvantages. Firstly, it limits the focusing optics to non-dispersive devices and sources to broad-spectrum X-ray sources. This implies some restrictions in costs, focal point size, beamline layout, beam intensity etc. Also, the total number of photons impinging on the sample during an experiment must be much higher than for a monochromatic experiment, as much of the spectrum is not used. This can also negatively affect the measurement time, as the heat load on the sample must be managed.

As synchrotron sources are getting brighter and 2D X-ray detectors are getting larger and faster, we show that it has become feasible to obtain accurate reciprocal-space maps of more than one Bragg reflection simultaneously, using a monochromatic nano-focused X-ray beam. This alignment of the sample in such a way that two Bragg reflections simultaneously satisfy the diffraction condition in a monochromatic beam is also called three-beam geometry. We proceed to show that with the incompressibility condition this suffices to obtain the full deformation tensor in any coordinates, notably those aligned with characteristic directions (e.g. facets) of the sample. As an example of the technique, an area of $8 \times 14 \mu \mathrm{m}$ was mapped with $150 \times 150 \mathrm{~nm}$ spatial resolution (beam size) showing the intrinsic strain in an as-grown $\mathrm{VO}_{2}$ microwire.

\section{Background and experiment}

The following two sections illustrate the calculations required to obtain the full deformation tensor in laboratory coordinates from the measurement of at least two Bragg reflections in a mutual projection. The following three sections concern the sample, sample alignment and actual experimental conditions.

\subsection{Components of a deformation tensor from a single Bragg} reflection

A monochromatic nano-diffraction experiment exploring the planes $(h k l)$ for a given position on the sample needs to measure the $2 \mathrm{D}$ scattered intensity in the $h k l$ Bragg reflection at that position for a set of angles [e.g. $\phi$ in Fig. 1(a)] around the reflection condition for the Bragg reflection. The sum intensity on the 2D detector expressed as a function of the sample rotation angle represents the rocking curve for that reflection, with its reflection at the scattering angle $2 \theta$ which fulfils the diffraction condition $\lambda=2 d_{h k l} \sin (\theta)$, where $\lambda$ and $d_{h k l}$ are the incoming wavelength and lattice spacing, respectively.

For each sample angle $\phi_{i}$ the 2D detector image can be interpreted as an accordingly oriented slice through the scattered intensity in reciprocal space. Thus, the whole Bragg reflection in reciprocal space can be reconstructed from multiple 2D diffraction intensity distributions, as shown in Fig. 1(b). Thereby, the reciprocal length of the scattering vector $|\mathbf{q}|=\left|\mathbf{k}_{\mathrm{o}}-\mathbf{k}_{\mathrm{i}}\right|=4 \pi \sin (\theta) / \lambda=2 \pi / d_{h k l}$ and its orientation in the laboratory system are measured. A change in the position of the Bragg reflection with respect to either an externally defined value or an internal norm can be described as a corresponding deformation of the set of planes $(h \mathrm{kl})$ investigated. We will use the mean value for all raster-scanned points as an internal $d_{\text {mean }}$ reference. The normal strain deformation along the direction perpendicular to the $(h k l)$ planes is then

$$
\epsilon_{\| h k l}=\epsilon_{q q}=\left|d-d_{\text {mean }}\right| / d_{\text {mean }} .
$$

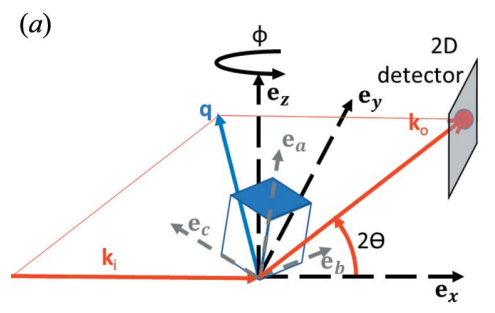

(b)

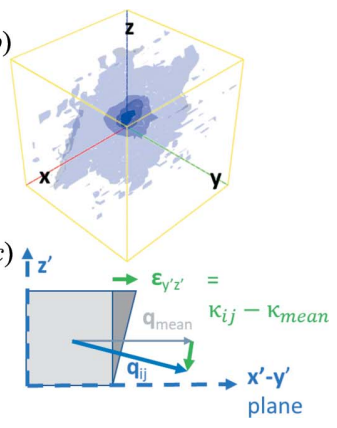

Figure 1

(a) Sketch showing coordinates for XRD mapping with $\mathbf{k}_{\mathrm{i}}, \mathbf{k}_{\mathrm{o}}$ and $\mathbf{q}$ the incoming, outgoing and scattering vectors, respectively, $\mathbf{e}_{x}, \mathbf{e}_{y}, \mathbf{e}_{z}$ the laboratory coordinates, and $\mathbf{e}_{a}, \mathbf{e}_{b}, \mathbf{e}_{c}$ the sample coordinates. The sample is aligned so that a diffraction reflection can be imaged on a $2 \mathrm{D}$ detector. In this orientation, the sample is raster-scanned in the $y z$ plane, perpendicular to the incoming X-rays $\mathbf{k}_{\mathrm{i}}$. The detector image for each sample-incoming $\mathrm{X}$-ray angle ( $\phi$ typically rotated around the $z$ axis) represents a slice of the $3 \mathrm{D}$ intensity distribution for the scattered X-rays $\mathbf{k}_{\mathrm{o}}$. Measuring multiple angles around the scattering condition allows the reconstruction of the scattering vector $\mathbf{q}$ in three dimensions, as shown in (b) for one point of the raster scan. The sketch (c) illustrates that when $\mathbf{q}$ is expressed in spherical coordinates the difference between its components and an expected (mean) value can be equated to the components of the deformation tensor in aligned Cartesian coordinates $\left(\mathbf{x}^{\prime} \mathbf{y}^{\prime} \mathbf{z}^{\prime}\right)$; 'pitch' $=\epsilon_{y^{\prime} z^{\prime}}=\epsilon_{\kappa \kappa}$ is illustrated. 
The position of the Bragg reflection can of course be expressed in arbitrary coordinate systems. By default we measure the position with respect to the laboratory coordinates $\mathbf{e}_{x}, \mathbf{e}_{y}, \mathbf{e}_{z}$ which, respectively, point along the incoming beam, vertically and horizontally, as shown in Fig. 1(a). These are easily transformed to spherical coordinates $\left(\mathbf{e}_{q}, \mathbf{e}_{\phi}, \mathbf{e}_{\kappa}\right)$, where $\mathbf{e}_{q}$ is aligned along the scattering vector, $\mathbf{e}_{\phi}$ rotates around the $z$ axis, in plane with respect to the $x y$ plane, and $\mathbf{e}_{\kappa}$, the polar angle, rotates out of plane with respect to the $x y$ plane:

$$
q=|\mathbf{q}|, \quad \phi=\arctan (x / y), \quad \kappa=\arcsin (z /|\mathbf{q}|) .
$$

As we are probing the small angular area around a Bragg reflection, we can linearize these to local Cartesian coordinates $\left(\mathbf{e}_{x^{\prime}}, \mathbf{e}_{y^{\prime}}, \mathbf{e}_{z^{\prime}}\right)$ :

$$
y^{\prime}=q-q_{\text {mean }}, \quad x^{\prime}=\phi-\phi_{\text {mean }}, \quad z^{\prime}=\kappa-\kappa_{\text {mean }} .
$$

Thus, the locally aligned coordinates express the small relative change of each component of the position of the Bragg reflection with respect to the mean value across the whole sample. We can associate these relative changes with the corresponding component of the deformation tensor, expressed in the same, primed coordinates, as shown in Fig. 1(c):

$$
\begin{array}{r}
\text { 'stretch' }=\epsilon_{y^{\prime} y^{\prime}}, \\
\text { 'yaw' }=\epsilon_{x^{\prime} y^{\prime}}, \\
\text { 'pitch' }=\epsilon_{y^{\prime} z^{\prime}} .
\end{array}
$$

For each Bragg reflection, we can thus calculate the components of the deformation tensor expressed in the local coordinates as defined by the mean position of the Bragg reflection (superscript):

$$
\left(\begin{array}{ccc}
\epsilon_{x^{\prime} x^{\prime}} & \boldsymbol{\epsilon}_{\mathbf{x}^{\prime} \mathbf{y}^{\prime}} & \epsilon_{x^{\prime} z^{\prime}} \\
\cdot & \boldsymbol{\epsilon}_{\mathbf{y}^{\prime} \mathbf{y}^{\prime}} & \boldsymbol{\epsilon}_{\mathbf{y}^{\prime} \mathbf{z}^{\prime}} \\
\cdot & \cdot & \epsilon_{z^{\prime} z^{\prime}}
\end{array}\right)^{q_{\text {mean }}, \phi_{\text {mean }}, \kappa_{\text {mean }}}
$$

The components printed in bold denote the known components: $\epsilon_{x^{\prime} y^{\prime}}, \epsilon_{y^{\prime} y^{\prime}}, \epsilon_{y^{\prime} z^{\prime}}$. The unknown and symmetric components are represented in normal print and by a simple dot, respectively.

\subsection{Combining Bragg reflections to obtain the full defor- mation tensor}

The defining property of tensors is that they do not change under a coordinate transform. It is possible to simultaneously measure two Bragg reflections and each Bragg reflection gives us three of the six independent components of the deformation tensor in the respectively aligned coordinate systems. By construction in Section 2.1, the respective components are expressed in local, primed coordinates. To combine both tensors we can express them in the mutual Cartesian basis $\left(\mathbf{e}_{x}, \mathbf{e}_{y}, \mathbf{e}_{z}\right)$ by coordinate transformation. We choose to mutually align the directions of $\mathbf{e}_{y^{\prime}}$, for which we know the normal strain, with the horizontal $\mathbf{e}_{y}$ axis. As we have the coordinates $q_{\text {mean }}$, the rotation matrix $R$ that corresponds to this alignment is on hand, but, because the calculation sets up the system of equations to be solved later, we find it instructive to illustrate it as a subsequent rotation around two axes in the following.

As shown in Fig. 2, we start with the original direction $\mathbf{e}_{q}$. First, rotate around the $z$ axis by the in-plane component of the scattering angle, $\phi$. Applying the corresponding rotation matrix $R_{z}$ to $\mathbf{e}_{q}$ gives us $\mathbf{e}^{\prime}$, a unit vector in the $y z$ plane. Next, we rotate around the $x$ axis by the out-of-plane component of scattering, $\kappa$, to get $R_{x}$. Now $\mathbf{e}^{\prime \prime}=R_{x} \mathbf{e}^{\prime}=R_{x} R_{z} \mathbf{e}_{q}$ is parallel to the $y$ axis. We can shorten this: $R=R_{x} R_{z}$, which is the expected rotation matrix. By this construction, the rotation by $R$ preserves the correct orientation of the other two coordinates so that $x^{\prime}$ transforms to $x$ and $z^{\prime}$ to $z$. See the supporting information for an explicit implementation of this operation in the programming language Python using the NumPy and SymPy libraries.

From the experimental data we can immediately calculate the two appropriate rotation matrices $R_{\text {red }}$ and $R_{\text {blue }}$, named by the colour used to indicate the region of interest on the detector image in Fig. 3. Next, we express the two deformation tensors in this mutual coordinate system and equate the components:

$$
\left(R_{\text {red }}^{\mathrm{T}} \epsilon_{\text {red }}^{\prime} R_{\text {red }}-R_{\text {blue }}^{\mathrm{T}} \epsilon_{\text {blue }}^{\prime} R_{\text {blue }}\right)_{i j}=0
$$

where $\epsilon_{\text {red }}^{\prime}$ and $\epsilon_{\text {blue }}^{\prime}$ denote the two deformation tensors as calculated above for the respective colour-coded regions on the detector. The deformation tensors expressed in rotated coordinates are still symmetric (see the supporting information); therefore, it may be tempting to believe that the above system of equations represents six linear equations for the six unknown components, three per Bragg reflection. Unfortunately, this is not the case, and in the supporting information we show explicitly for a random list of scattering angles that the determinant of the equation matrix is zero. One can also understand intuitively that there is no information in the measured data for one of the normal strain components. Consider the case where the two measured scattering vectors
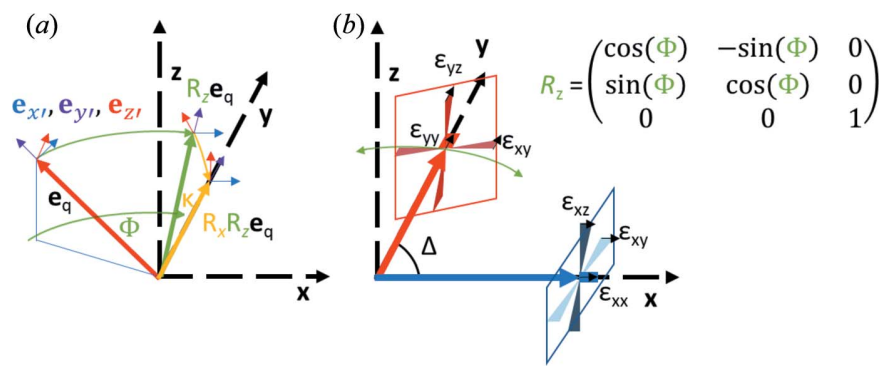

Figure 2

(a) This illustrates the calculation of the coordinate transformation from the Cartesian coordinates aligned along the scattering vector to laboratory coordinates. The initial local coordinates $\mathbf{e}_{x^{\prime}}, \mathbf{e}_{y^{\prime}}, \mathbf{e}_{z^{\prime}}$ are shown as small blue, purple and red arrows which are transformed in parallel. They follow as the unit vector $\mathbf{e}_{q}$ is rotated first around the $z$ axis by $\phi$ giving $R_{z} \mathbf{e}_{q}$, shown in green, to lie in the $y z$ plane. Then a rotation around the $x$ axis by $\kappa$ aligns $R_{x} R_{z} \mathbf{e}_{q}$ (yellow) and $R_{x} R_{z} \mathbf{e}_{y^{\prime}}$ with the $y$ axis, $R_{x} R_{z} \mathbf{e}_{x^{\prime}}$ with the $x$ axis and $R_{x} R_{z} \mathbf{e}_{z^{\prime}}$ with the $z$ axis. The sketch $(b)$ shows the known components of the deformation tensor for the special case that the blue and red scattering vectors are parallel to the $x$ and $y$ axes, respectively. Note that now $\epsilon_{z z}$ remains unknown. 
both lie in the $x y$ plane, each parallel to one of the axes, as sketched in Fig. 2(b). Now it is apparent that the deformation tensor component $\epsilon_{x y}=\epsilon_{y x}$ is measured twice and that the $\epsilon_{z z}$ component is not measured at all. Mutual alignment in this case is only rotation around the $z$ axis, explicitly performed by $R_{z}$, which only mixes the known components. The sketched case can be generalized to any arbitrary angle $\Delta$ between the scattering vectors. Note that the underdetermined case, where $\Delta=0$ or $180^{\circ}$, is physically impossible for scattering vectors. Therefore, in the set of the six linear equations above, there is exactly one that can be expressed as a linear combination (as a function of $\Delta$ ) of the others. We thus need to find a further constraint to the deformation and naturally choose linear incompressibility:

$$
\epsilon_{x x}+\epsilon_{y y}+\epsilon_{z z}=0
$$

which is generally well preserved in hard condensed matter and crystals without pores, such as the present case of a freestanding microwire. Now, solving for the unknown components of both deformation tensors and expressing either in the laboratory coordinates gives the desired full deformation tensor.

Finally, from the data acquired in Section 2.4 (see below), the relative orientation of the sample regarding the laboratory coordinates will be known (see also the supporting information). We can thus calculate the components of the deformation tensor for coordinates aligned to the sample facets $\left(\mathbf{e}_{a}, \mathbf{e}_{b}, \mathbf{e}_{c}\right)$, as shown in Fig. 2. We find a rotation matrix

$$
R_{a b c \rightarrow x y z}=\left(\begin{array}{ccc}
-0.04893713 & -0.16695799 & 0.9847488 \\
0.83148366 & -0.55306786 & -0.05244857 \\
0.55338955 & 0.81623584 & 0.16588841
\end{array}\right) .
$$
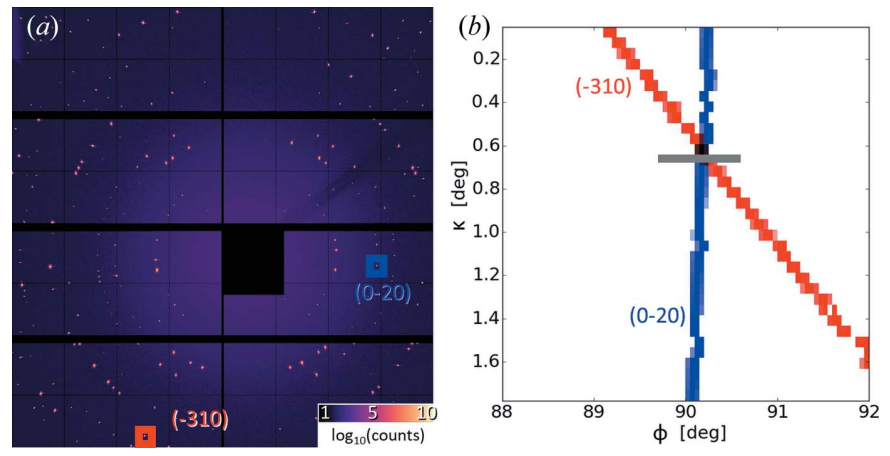

Figure 3

(a) Integrated intensity of all diffraction patterns taken on the 2D X-ray detector, while the $\mathrm{VO}_{2}$ microwire was rotated at fixed $\kappa$ by $270^{\circ}$ around $\phi$. To facilitate reflection detection, a large region in the centre of the detector, around the shadow of the beam-stop, is masked. All visible reflections were successfully indexed (see Fig. S1) and the two regions of interest marked in blue and red correspond to the Bragg reflections $(0 \overline{2} 0)$ and $(\overline{3} 10)$, respectively. The intensity measured in these regions is plotted for a raster scan of the sample orientation angles $\phi$ and $\kappa$ in $(b)$. The intensities form lines representing the respectively fulfilled diffraction condition. At the crossing point the grey line indicates the angular range in $\phi$ used for the following measurement.
The detailed calculations can be found in the supporting information.

\subsection{The sample}

The material under investigation here is vanadium dioxide. It shows a reversible phase transition at about $341 \mathrm{~K}$ (Liu et al., 2011). The material changes from a low-temperature insulating phase to a high-temperature metallic phase. Many material properties such as the dielectric properties and conductivity are abruptly changed. This has potential applications for smart optical components (Rensberg et al., 2016) and window coatings (Zhou et al., 2013; Chen et al., 2011). It is known that strain modifies the transition temperature (Cao et al., 2009; Cao \& Wu, 2011; Aetukuri et al., 2013), a key requirement for the technological application of this material.

The sample investigated in this work is an as-grown $\mathrm{VO}_{2}$ microwire, grown from $\mathrm{V}_{2} \mathrm{O}_{5}$ powder on a rough quartz substrate as described in the work of Cheng et al. (2012). The wires grow in the rutile high-temperature phase, but we will reference all crystallographic orientations in the monoclinic phase, as present during the experiment, at room temperature. The microwires have a rectangular base, facetted at $\{011\}$ and $\{01 \overline{1}\}$, and the principal axis is oriented along (201) (Wang et al., 2018). These three orthogonal directions will be our reference coordinate system for the deformation tensor. Note that in monoclinic $\mathrm{VO}_{2}(20 \overline{1})$ is almost parallel to [201]], because the structure has only a slight distortion of the rutile crystal.

A single microwire with a length $>100 \mu \mathrm{m}$ was removed from the growth substrate and one end was glued onto the finely pulled point of a glass microcapillary using a very small drop of two-component epoxy. The freestanding end of the microwire was investigated using the ID13 microfocus X-ray beamline at the ESRF Grenoble. The capillary was mounted and centred on the home-built goniometer at ID13, as shown in Fig. S2 (in the supporting information).

\subsection{Sample alignment}

To arrive at the diffraction condition for a given plane $(h k l)$ and X-ray wavelength $\lambda$, it is necessary to correctly orient the sample in three dimensions. A large variety of goniometers are available for this task. Generally, the angles of rotation can be decomposed into two orthogonal components $\phi, \kappa$. The goniometer used here allows for a full rotation around the axis $\phi$, which can be tilted by an underlying cradle stage to an angle $\kappa$ with respect to the laboratory $z$ axis. As the wire is rotated in an initially unknown way about its principal axis, a preliminary scan to find its orientation was performed. The wire was rotated for $270^{\circ}$ around $\phi$ and a diffractogram was measured every $0.1^{\circ}$ of rotation. The wire was scanned laterally for each projection to ensure it was illuminated.

The individual reflections were identified and indexed. The accumulated and selected indexed data of this scan are shown in Fig. 3(a); the fully indexed frame can be found in Fig. S1. The analysis confirms that the microwire was in the lowtemperature $\mathrm{VO}_{2}$ monoclinic structure with $a^{*}=5.75$, 
$b^{*}=4.53, c^{*}=5.39 \mathrm{~nm}^{-1}, \alpha=90.0, \beta=122.6, \gamma=90.0^{\circ}$, in close agreement with the expected structure (Inorganic Crystal Structure Database 34033).

Fig. 3(b) indicates how to find an orientation of the sample where the diffraction condition for two Bragg reflections is simultaneously fulfilled. Depending on the crystal symmetry and incoming X-ray wavelength, it can be shown by an Ewald sphere construction that there are plenty of such crossing points of fulfilled diffraction conditions [see the supporting information and James (1982)]. Many high-symmetry points even show more than two crossing points in very close proximity.

It must be noted that the diffraction at one set of planes can be influenced by the fact that a second set of planes also fulfils a diffraction condition. Strictly speaking, the scattering process cannot be treated kinematically as a superposition of two independent scattering events, but must be treated dynamically, considering the wavefield in the periodic potential of the crystal. The threshold for relevant deviations from the kinematic approximation is found where the scattered intensity approaches the same order of magnitude as the incoming beam intensity. However, for the present case of a very small sample $(\sim \mu \mathrm{m})$, small scattering cross section and large scattering angles, we are very far from this case.

The rotational scan data were used to calculate the sample orientation with respect to laboratory coordinates (see the supporting information). This relation will be very useful to calculate the deformation tensor components in coordinates aligned to the sample facets. For simplicity, we choose to call the wire axis $\mathbf{e}_{a}$, the normal to the (01) facet $\mathbf{e}_{b}$ and the normal to the (011) facet $\mathbf{e}_{c}$, to obtain a Cartesian coordinate system aligned to the sample facets $\left(\mathbf{e}_{a}, \mathbf{e}_{b}, \mathbf{e}_{c}\right)$. It is most useful to express the components of the deformation tensor in these coordinates, which are naturally aligned with possible stresses in the sample, rather than in the arbitrarily aligned laboratory coordinates.

\subsection{Summary of experimental conditions}

The diffraction experiments were performed at the ID13 microfocus beamline with a $15.20 \mathrm{keV}$ X-ray beam focused down to $150 \times 150 \mathrm{~nm}$. The divergence of the beam was around $4 \mathrm{mrad}$ due to the focusing. The detector was a Dectris Eiger4M with $2167 \times 2070$ pixels of size $75 \times 75 \mu \mathrm{m}$. The sample-detector distance was $127.53 \mathrm{~mm}$, calibrated using $\mathrm{Al}_{2} \mathrm{O}_{3}$ and pyFAI (Kieffer \& Karkoulis, 2013).

The sample was mounted on a home-built two-axis goniometer using SmarAct piezo stages. A base cradle stage (SGO-60.5) rotated by $\kappa$ around the laboratory $y$ axis and a rotary stage (SR-2013) rotated by $\phi$ around the laboratory $z$ axis when the base is at $\kappa=0$. The goniometer was completed by two, crossed linear stages (SLC-1720) used to reduce the sphere of confusion by following a look-up table of corrections for rotation around the $z$ axis. (Refer to Fig. S2 for a photograph of the setup.) The goniometer was mounted on a PI MARS $x y z$ piezo stage, which performed the lateral scans in laboratory $y$ and $z$ directions, as the fast and slow axis, respectively.
The scanning parameters were $y, z, \phi: 8 \mu \mathrm{m} \times 14 \mu \mathrm{m} \times 0.9^{\circ}$ in $80 \times 140 \times 26$ points. The piezo $y$ axis was scanned continuously using the recently developed BLISS beamline control system (Guijarro et al., 2018). The detector was read out every $25 \mathrm{~ms}$ with a negligible readout time of $10 \mu \mathrm{s}$. These time steps correspond to $100 \mathrm{~nm}$ steps in the direction of travel. The $z$ axis was raster-scanned in $100 \mathrm{~nm}$ steps, followed by the angle $\phi$. The total exposure time was thus a little more than $2 \mathrm{~h}$. In practice, there is a little overhead incurred during the return stroke of the $y$ axis and the rotation, and thus the full mapping took $2.5 \mathrm{~h}$.

During all scans an X-ray fluorescence (XRF) detector recorded fluorescence of the vanadium $K$ line, which was used to find any drift in vertical position for each map. After vertical correction, the horizontal offset for every line of every scan was corrected (see Fig. S4). As the outline of the microwire in the fluorescence signal is an unmistakable and sharp reference, this ensured true overlap of the maps for each angle at a one-pixel (100 nm) accuracy.

The diffraction data were analysed for each point of the $3 \mathrm{D}$ real-space map $(y, z, \phi)$ by first extracting a generous region of interest in the detector for each of the two Bragg reflections. This greatly reduces the data-set size. The diffraction data were then remapped to the corrected real-space positions using the corrections measured by XRF and linear interpolation. Next, the data were transposed and integrated in reciprocal laboratory coordinates using xrayutilities (Kriegner et $a l ., 2013)$. Finally, the centre of mass was calculated in spherical coordinates, to give the scattering vector in the required form. The map of these values is the input for the calculations outlined earlier and in the supporting information.

\section{Results}

The diffraction results can be interpreted as the integral of the diffracted intensity as the incoming X-ray beam traverses the microwire. Note, for small samples and large beam coherence, this approximation can fail spectacularly as coherent effects appear. Working under the incoherent assumption, the results represent the average of all the deformation contributions along the beam's path. As shown in the sketch at the left of Fig. 4 , the $2 \mathrm{D}$ raster map produces a projected view cutting skew through the microwire's cross section. The sketch was aligned by hand to the features resembling the edges of the rectangular volume of the microwire in deformation tensor components. These features are most notable in the maps showing $\epsilon_{b b}, \epsilon_{c c}$ and $\epsilon_{b c}$. The resulting alignment agrees well with the alignment of the sample found in Section 2.4 and Fig. S3.

The diagonal components of the deformation tensor correspond to the normal strain deformation of the material. In Fig. 4 one can see that as the wire forms a tip it is increasingly compressed along the axial, $a$, direction. As imposed by the analysis and expected for an incompressible material, the compression along one axis is compensated in the other two. Interestingly, this compensation appears much stronger along the $c$ direction than $b$. A strong interplay 


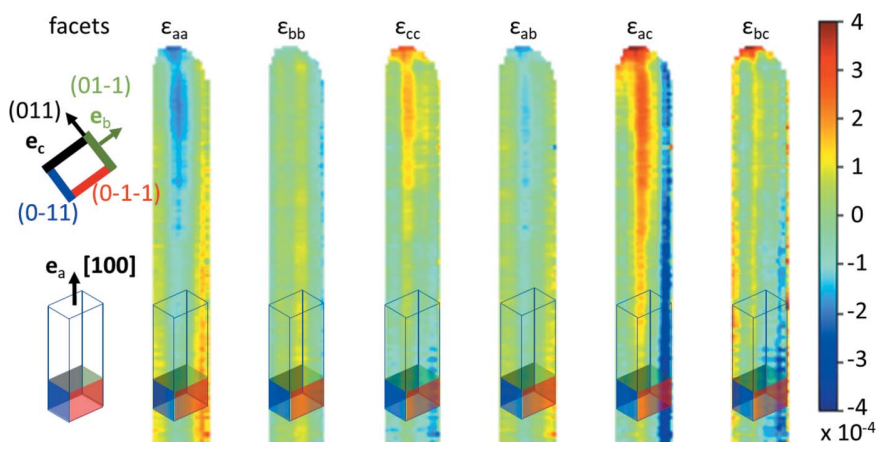

Figure 4

Plot of the calculated components of the deformation tensor expressed in coordinates aligned to the microwire facets $(\mathbf{a}, \mathbf{b}, \mathbf{c})$. The lower inset sketch illustrates the orientation of the facets $\mathbf{c}, \mathbf{b},-\mathbf{c}$ and $-\mathbf{b}$ in black, green, red and blue, respectively. The sketch is superposed onto the raster-scanned map to guide the eye.

between these two axes is also apparent in that the largest shear component is $\epsilon_{a c}$. This is attributed to the large anisotropy of the mechanical properties of monoclinic $\mathrm{VO}_{2}$ (Gaillac et al., 2016).

Finally, a faint lattice expansion on the faces of facets in $\epsilon_{b b}$ and $\epsilon_{c c}$ is contrasted by a relative compression on the edges. With the shear in $\epsilon_{b c}$ this may indicate a slight rounding of the rectangular shape, which could be traced back to the minimization of the surface energy.

\section{Discussion}

In the following we will try to outline the main factors in arriving at an upper bound on the errors of the presented method.

\subsection{Measurement error}

Firstly, the edges of the final data voxels were longer than the projected width and height of the detector pixels, but smaller than $1 / 3$ of the width of the Bragg reflection. The angular range of the scans in $\phi$ map the scattered intensity down to $1 \times 10^{-3}$ in both directions. The 3D Bragg reflections were evaluated by the centre of mass in three dimensions. The minimum number of counts on the detector per reflection was $1 \times 10^{7}$ and the FWHM of the reflection $1 \times 10^{-3}$ (relative units), so that the statistical error of this estimator is $<1 \times 10^{-6}$ and thus negligible. In the measurement geometry presented here, the measured Bragg reflections did not impinge normally onto the detector. Therefore, an error in the sample orientation $\phi$ and $\kappa$ affects the position in reciprocal space of all three coordinates of the Bragg reflection. Using a laser reflection setup, the accuracy of both encoded rotational stages was estimated to be better than $1 \times 10^{-4} \circ$. It must be noted that this value is of course critically dependent on the sturdy attachment of the sample to the stage, especially under the excited conditions of a lateral raster scan.

An additional factor to consider is refraction at the facets of the microwire (Kriegner et al., 2011). Fortunately, the refractive index of $\mathrm{VO}_{2}$ for $\mathrm{X}$-rays at $15 \mathrm{keV}$ differs from 1 only by
$1 \times 10^{-5}$, leading to negligible changes in the scattering angles in the presented data. However, for heavier materials and lower X-ray energies, this contribution may become significant.

For a sample larger than the beam size, the width of the diffraction reflection is mainly determined by the divergence of the incoming, nano-focused X-ray beam at roughly $0.4 \mathrm{mrad}$ or $0.02^{\circ}$. Finally, the accuracy of the measured parameters is influenced by the angular sampling step size, in this case $0.03^{\circ}$. To optimize measurement time, this was chosen to be approximately equal to the width of the Bragg reflection. As is, it remains the largest single contribution to measurement uncertainty. For the scattering angle of $35^{\circ}$ this naively corresponds to a relative measurement error of $2 \times 10^{-4}$. However, as the diffractograms are analysed as an ensemble after projection into and resampling in reciprocal space, the final error in the centre of mass calculation is somewhat lower. Also, depending on the specific orientation of the scattering vector, this error affects the various components unequally.

In summary, we conclude that the measurement of the Bragg reflection coordinates we performed has a relative uncertainty of around $1 \times 10^{-4}$. As the components of the deformation tensor are equal to the normalized difference between the error-laden measured value and the fixed mean value, this relative uncertainty in the Bragg coordinate represents the absolute uncertainty of the deformation tensor coordinate in local coordinates. We finally calculated the standard error progression by partial derivatives of the deformation tensor components with respect to the measured variables, multiplied by the respective uncertainty and summed. This gives an estimate for the experimental uncertainty of around $2 \times 10^{-4}$ for all the deformation tensor components.

\subsection{Fit error}

We obtain the components of the deformation tensor in the sample coordinate system, by rotation of the coordinate systems for two generally directed Bragg reflections. We arrived at an overdetermined set of equations which we solved via an explicit least-squares fit (LSE, see the supporting information). In Fig. 2 one can see that the expression of the Bragg reflections in a mutual coordinate system involves projecting the components onto the new axes. With the LSE in mind, the phase space is filled most evenly with Bragg reflections evenly dispersed in 3D space. Two Bragg reflections should ideally be separated by an angle of $90^{\circ}$. Fortunately, the three-beam geometry excludes the underdetermined cases of 0 and $180^{\circ}$, and generally this problem is well conditioned. For the present case the angle between the two scattering vectors is $110^{\circ}$.

The LSE fitting directly provides an estimation for the standard error of the fitted parameters, which again can be inserted in the error propagation evaluation chain. Now the standard error is a function of the measured parameters, as their spread is a measure for the goodness of the fit. As seen in Fig. 5, there is large spread in the values for the standard error 


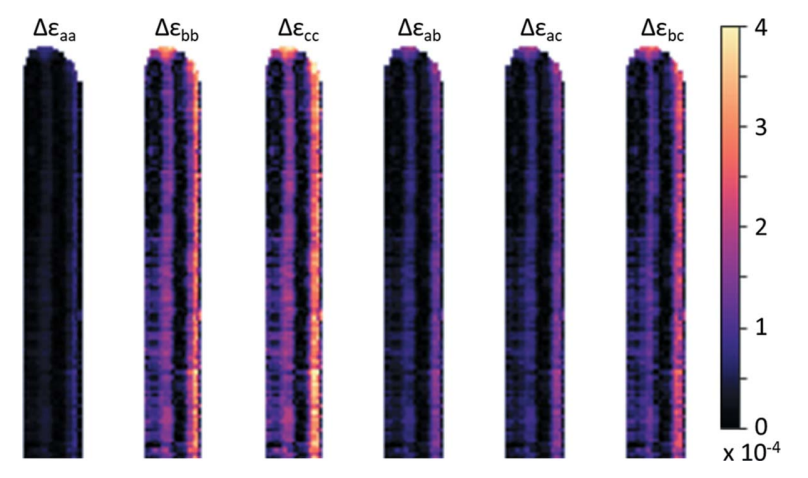

Figure 5

Plots of the standard error after the least-squares fit for the components of the deformation tensor $\epsilon$ expressed in coordinates aligned with the sample facets $(\mathbf{a}, \mathbf{b}, \mathbf{c})$. The width of the microwire projection is $2 \mu \mathrm{m}$.

across the resultant variables and the mapped spatial coordinates.

\subsection{Reliability of results}

A quick back-of-the-envelope calculation shows the order of magnitude for surface tension induced deformation:

$$
\epsilon=\frac{\sigma}{Y}
$$

where $Y$ is Youngs modulus and $\sigma$ the surface tension induced stress. For $\sigma$ we insert

$$
\sigma=4 \frac{d}{A} \mathrm{SE},
$$

with $d=1 \mu \mathrm{m}$ the side facet width, of which there are four; $A=$ $1 \mu \mathrm{m}^{2}$, the area of the top of the rectangular microwire; and surface energy $(\mathrm{SE})=1 \mathrm{~J} \mathrm{~m}^{-2}$ (Wang et al., 2018). As mentioned, the mechanical properties of monoclinic $\mathrm{VO}_{2}$ are anisotropic, but for a very realistic value of $40 \mathrm{GPa}$ (Gaillac et al., 2016), we obtain $\epsilon=1 \times 10^{-4}$.

Comparing the magnitude of the measured deformation tensor components with the estimated measurement error, we see clearly that the observed effects are on the cusp of resolvability. An argument can be made that measurement errors are unlikely to correlate to the structure of the microwire. However, as outlined in the previous discussion, the facet surfaces and sample orientation drift are both sources of systematic errors. We have tried to avoid their influence, but they represent a certain risk for misinterpretation of results obtained by the method outlined in this study and they must be carefully considered for each sample and measurement geometry.

\section{Conclusion and outlook}

We have outlined a method to map the full deformation tensor for a micro-sized crystal. Compared with strain maps obtained by analysing a single Bragg reflection, the presented method reveals a more complete picture of the actual deformed state of the sample. As XRD is already a well-established method for strain analysis and 2D detectors are becoming larger, faster and cheaper, we predict that the three-beam geometry, simultaneously measuring two Bragg reflections, will become more and more popular.

As outlined, we obtain a $2 \mathrm{D}$ projection of the deformation within the sample volume. This can be viewed as the average contribution of all differently deformed sub-volumes along the path of the incoming X-ray beam. We also showed that for the present sample symmetry and X-ray energy there are many sample orientations where the diffraction conditions for two Bragg reflections are simultaneously fulfilled. With enough projections, the distribution in the 3D sample volume of each component of the deformation tensor can be revealed, for example by filtered back-projection. As we have shown, the measurement times are sufficiently fast to make this feasible. Considering the large penetration depth of X-rays, and the important role micro- and nano-structured crystals play in modern technology, this outlook opens a plethora of possible studies, adding to the large number of possible correlative studies (Ulvestad et al., 2019).

When envisioning 3D sample reconstructions, we must compare our method with Bragg ptychography. The method we present is based on straightforward XRD nano-diffraction and as such does not rely on any coherent beam profile and sample reconstruction. Only the centre of mass of the Bragg reflection must be accurately measured for each point. Therefore, it is much more stable with respect to changes in the beam, allowing a much faster scanning and a much larger field of view. Importantly, any sample drift can be easily corrected post-measurement. The centre of mass estimation is already accurate with very few detected photons compared with the detailed reconstruction of the Bragg reflection required for Bragg ptychography. The drawback is, of course, that many projections are required to arrive at $3 \mathrm{D}$ information and that there is no sub-beam size resolution.

These points aside, the presented calculation using two Bragg reflections measured simultaneously can also be applied to Bragg ptychography experiments. To date, these typically limit themselves to a single reflection, yet the information obtained per Bragg reflection is a $3 \mathrm{D}$ volume of the same parameters we have measured here in two dimensions. Therefore, when two or more Bragg reflections are measured by ptychography, the calculations outlined in this work can be performed voxel-wise to gain the full deformation tensor in three dimensions.

\section{Acknowledgements}

The authors acknowledge the work of the ESRF Beamline Control Unit and the BLISS Development Team. In particular, the excellent support by R. Homs, M. Guijarro, C. Guilloud and S. Petitdemange before and during the experiment is much appreciated.

\section{References}

Abboud, A., Kirchlechner, C., Keckes, J., Conka Nurdan, T., Send, S., Micha, J. S., Ulrich, O., Hartmann, R., Strüder, L. \& Pietsch, U. (2017). J. Appl. Cryst. 50, 901-908.

Aben, H., Anton, J., Õis, M., Viswanathan, K., Chandrasekar, S. \& Chaudhri, M. M. (2016). Appl. Phys. Lett. 109, 231903. 
Aetukuri, N. B., Gray, A. X., Drouard, M., Cossale, M., Gao, L., Reid, A. H., Kukreja, R., Ohldag, H., Jenkins, C. A., Arenholz, E., Roche, K. P., Dürr, H. A., Samant, M. G. \& Parkin, S. S. P. (2013). Nat. Phys. 9, 661-666.

Cao, J., Ertekin, E., Srinivasan, V., Fan, W., Huang, S., Zheng, H., Yim, J. W. L., Khanal, D. R., Ogletree, D. F., Grossman, J. C. \& Wu, J. (2009). Nat. Nanotechnol. 4, 732-737.

Cao, J. \& Wu, J. (2011). Mater. Sci. Eng. Rep. 71, 35-52.

Chen, Z., Gao, Y., Kang, L., Du, J., Zhang, Z., Luo, H., Miao, H. \& Tan, G. (2011). Solar Energy Mater. Solar Cells, 95, 2677-2684.

Cheng, C., Liu, K., Xiang, B., Suh, J. \& Wu, J. (2012). Appl. Phys. Lett. 100, 103111.

Chung, J.-S. \& Ice, G. E. (1999). J. Appl. Phys. 86, 5249-5255.

Gaillac, R., Pullumbi, P. \& Coudert, F.-X. (2016). J. Phys. Condens. Matter, 28, 275201.

Guijarro, M., Beteva, A., Coutinho, T., Dominguez, M.-C., Guilloud, C., Homs, A., Meyer, J., Michel, V., Papillon, E., Perez, M. \& Petitdemange, S. (2018). Proceedings of the 16th International Conference on Accelerator and Large Experimental Control Systems (ICALEPCS2017). JACoW Publishing.

James, R. W. (1982). The Optical Principles of the Diffraction of $X$-rays. Woodbridge: Ox Bow Press.

Kieffer, J. \& Karkoulis, D. (2013). J. Phys. Conf. Ser. 425, 202012.

Kriegner, D., Wintersberger, E., Kawaguchi, K., Wallentin, J., Borgström, M. T. \& Stangl, J. (2011). Nanotechnology, 22, 425704.
Kriegner, D., Wintersberger, E. \& Stangl, J. (2013). J. Appl. Cryst. 46, $1162-1170$.

Lee, M. L., Fitzgerald, E. A., Bulsara, M. T., Currie, M. T. \& Lochtefeld, A. (2005). J. Appl. Phys. 97, 011101.

Liu, W.-T., Cao, J., Fan, W., Hao, Z., Martin, M. C., Shen, Y. R., Wu, J. \& Wang, F. (2011). Nano Lett. 11, 466-470.

Rensberg, J., Zhang, S., Zhou, Y., McLeod, A. S., Schwarz, C., Goldflam, M., Liu, M., Kerbusch, J., Nawrodt, R., Ramanathan, S., Basov, D. N., Capasso, F., Ronning, C. \& Kats, M. A. (2016). Nano Lett. 16, 1050-1055.

Robach, O., Micha, J.-S., Ulrich, O., Geaymond, O., Sicardy, O., Härtwig, J. \& Rieutord, F. (2013). Acta Cryst. A69, 164-170.

Tardif, S., Gassenq, A., Guilloy, K., Pauc, N., Osvaldo Dias, G., Hartmann, J.-M., Widiez, J., Zabel, T., Marin, E., Sigg, H., Faist, J., Chelnokov, A., Reboud, V., Calvo, V., Micha, J.-S., Robach, O. \& Rieutord, F. (2016). J. Appl. Cryst. 49, 1402-1411.

Thibault, P., Dierolf, M., Menzel, A., Bunk, O., David, C. \& Pfeiffer, F. (2008). Science, 321, 379-382.

Ulvestad, A., Hruszkewycz, S. O., Holt, M. V., Hill, M. O., CalvoAlmazán, I., Maddali, S., Huang, X., Yan, H., Nazaretski, E., Chu, Y. S., Lauhon, L. J., Rodkey, N., Bertoni, M. I. \& Stuckelberger, M. E. (2019). J. Synchrotron Rad. 26, 1316-1321.

Wang, L., Ren, H., Chen, S., Chen, Y., Li, B., Zou, C., Zhang, G. \& Lu, Y. (2018). Cryst. Growth Des. 18, 3896-3901.

Zhou, J., Gao, Y., Zhang, Z., Luo, H., Cao, C., Chen, Z., Dai, L. \& Liu, X. (2013). Sci. Rep. 3, 3029. 